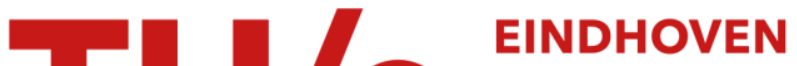 UNIVERSITY OF TECHNOLOGY
}

\section{Stability analysis of networked control systems : a sum of squares approach}

\section{Citation for published version (APA):}

Bauer, N. W., Maas, P. J. H., \& Heemels, W. P. M. H. (2012). Stability analysis of networked control systems : a sum of squares approach. Automatica, 48(8), 1514-1524. https://doi.org/10.1016/j.automatica.2012.05.023

DOI:

10.1016/j.automatica.2012.05.023

Document status and date:

Published: 01/01/2012

\section{Document Version:}

Accepted manuscript including changes made at the peer-review stage

\section{Please check the document version of this publication:}

- A submitted manuscript is the version of the article upon submission and before peer-review. There can be important differences between the submitted version and the official published version of record. People interested in the research are advised to contact the author for the final version of the publication, or visit the $\mathrm{DOI}$ to the publisher's website.

- The final author version and the galley proof are versions of the publication after peer review.

- The final published version features the final layout of the paper including the volume, issue and page numbers.

Link to publication

\section{General rights}

Copyright and moral rights for the publications made accessible in the public portal are retained by the authors and/or other copyright owners and it is a condition of accessing publications that users recognise and abide by the legal requirements associated with these rights.

- Users may download and print one copy of any publication from the public portal for the purpose of private study or research.

- You may not further distribute the material or use it for any profit-making activity or commercial gain

- You may freely distribute the URL identifying the publication in the public portal.

If the publication is distributed under the terms of Article 25fa of the Dutch Copyright Act, indicated by the "Taverne" license above, please follow below link for the End User Agreement:

www.tue.nl/taverne

Take down policy

If you believe that this document breaches copyright please contact us at:

openaccess@tue.nl

providing details and we will investigate your claim. 


\title{
Stability Analysis of Networked Control Systems: A Sum of Squares Approach
}

\author{
N.W. Bauer, P.J.H. Maas, W.P.M.H. Heemels \\ Dept. of Mechanical Engineering, Eindhoven University of Technology, P.O. Box 513, 5600 MB Eindhoven, the Netherlands
}

\begin{abstract}
This paper presents a sum of squares (SOS) approach to the stability analysis of networked control systems (NCSs) incorporating bounded time-varying delays, bounded time-varying transmission intervals and a shared communication medium. A shared communication medium imposes that per transmission only one node, which consists of several actuators or sensors, can access the network and transmit its corresponding data. Which node obtains access is determined by a network protocol. We will provide mathematical models that describe these NCSs and transform them into suitable hybrid systems formulations. Based on these hybrid systems formulations we construct Lyapunov functions using SOS techniques that can be solved using LMIbased computations. This leads to several beneficial features: (i) we can deal with plants and controllers which are described by nonlinear (piecewise) polynomial differential equations, (ii) we can allow for non-zero lower bounds on the delays and transmission intervals in contrast with various existing approaches, (iii) we allow more flexibility in the Lyapunov functions thereby obtaining less conservative estimates of the maximal allowable transmission intervals (MATI) and maximal allowable delay (MAD), and finally (iv) it provides an automated method to address stability analysis problems in NCS. Several numerical examples illustrate the strengths of our approach.
\end{abstract}

Key words: Networked Control Systems, Hybrid Systems, Sum of Squares

\section{Introduction}

Stability of networked control systems (NCSs) received considerable attention in recent years and several approaches are currently available for tackling this challenging problem. A distinction between the various approaches can be made on the basis of the modeling setup being adopted, the types of plants and controllers being allowed, and the network-induced imperfections being covered. In particular, the network-induced imperfections can roughly be divided in the following five categories: (i) Quantization errors in the signals transmitted over the network due to the finite word length of the

Email addresses: n.w.bauer@tue.nl (N.W. Bauer), p.j.h.maas@student.tue.nl (P.J.H. Maas), w.m.p.h.heemels@tue.nl (W.P.M.H. Heemels).

1 This work is partially supported by the Innovational Research Incentives Scheme under the VICI grant "Wireless control systems: A new frontier in automation" awarded by NWO (Netherlands Organization for Scientific Research) and STW (Dutch Science Foundation), the European 7th Framework Network of Excellence "Highly-complex and networked control systems (HYCON2)", and by the European Community through the FP7-ICT-2007-2 thematic program under the WIDE-224168 project. packets; (ii) Packet dropouts caused by the unreliability of the network; (iii) Variable sampling/transmission intervals; (iv) Variable communication delays (being smaller or larger than the transmission interval); (v) A shared communication medium imposing that only one node consisting of several actuators and sensors is allowed to transmit its information per transmission. The latter requires the presence of a network protocol that schedules which node gets access to the network at a transmission instant. In the analysis of NCSs exhibiting one or more of these networked-induced imperfections, several research lines can be distinguished.

A first line of research that can be distinguished is the discrete-time modeling approach, see e.g. [6-8,11,12,16, 18,34-36], which applies to linear plants and linear controllers and is based on exact discretization of the NCS between two transmission times. The resulting discretetime linear model in which the uncertain parameters such as the delay and transmission interval appear in an exponential form are overapproximated by polytopic models in which new uncertain parameters are introduced that appear in an affine form. These models are amendable for robust stability analysis methods based on linear matrix inequalities (LMIs). The most general 
discrete-time modeling approaches, in the sense of including the largest number of the above network-induced imperfections, are [6] including (ii), (iii) and (iv) (small and large delays), and [8] (ii), (iii) and (v) (only allowing so-called periodic and quadratic protocols), where (iv) with small delays can be easily included. Interestingly, in [8] the controllers can be both in a continuoustime or discrete-time form. Based on these models any of the available overapproximation techniques [16] can be applied to obtain polytopic models. In [16] a comparison is presented between the different overapproximation methods and the subsequent LMI-based stability analysis.

A second line of research on NCSs adopts the sampleddata modeling approach, which uses continuoustime models that describe the NCS dynamics in the continuous-time domain (so without exploiting any form of discretization) and perform stability analysis based on these sampled-data NCS models directly, see e.g. $[9,10,38,39]$. The models are in the form of delay-differential equations (DDEs) and LyapunovKrasovskii-functionals are used to assess stability based on LMIs. In [21] it is shown that the use of such an approach for digital control systems neglects the piecewise constant nature of the control signal due to the zero-order-hold mechanism thereby introducing conservatism when exploiting such modeling for stability analysis. This spurred the development of an alternative approach, recently proposed in $[23,24]$, which is based on impulsive DDEs that do take into account the piecewise constant nature of the control signal. Constructive LMI-based stability conditions in the latter line of work apply for linear plants and linear controllers. These LMI conditions cannot exploit the availability of non-zero lower bounds on transmission intervals and delays in the sense that they always apply to the case where the minimum delay and transmission interval are equal to zero. This approach includes (ii)-(iv) as network-induced imperfections.

A third line of research is formed by the continuous-time modeling (or emulation) approaches, which are inspired by the work in [37], and extended in [3, 4, 15, 25-27] and the recent work [14] that includes all five of the mentioned communication imperfections albeit under some restrictive assumptions. To describe the NCS, this research line exploits the hybrid modeling formalisms as advocated in [13]. The stability of the resulting hybrid system model is based on Lyapunov functions constructed by combining separate Lyapunov functions for the network-free closed-loop system (which has to be designed to satisfy certain stability properties) on the one hand and the network protocol on the other hand (or, alternatively, adopting directly small gain arguments). General network protocols are allowed and the plant and controller in this context can also be of a general nonlinear form, but have to be given as continuous-time differential equations. In addition $\mathcal{L}_{p}$ performance analy- sis $[15,26]$ can be easily incorporated in this framework. The available stability conditions all apply only for the case of zero lower bounds on the transmission intervals and delays.

In this paper we propose an alternative computational method for stability analysis of NCSs, which from a modeling point of view is closest to the continuous-time modeling approach as just discussed, although it includes also the models based on impulsive DDEs [23, $24,36]$, see Remark 2 in the main text. In particular, we will consider here NCSs that exhibit varying transmission intervals, varying delays and a shared communication medium. In fact, a preliminary version of our approach, applying to the case where the communication medium is not shared, is presented in [1]. In this extended version, we adopt rather general NCS models including a shared communication medium. These models will be converted into hybrid systems formulations [13]. Assuming piecewise polynomial plant dynamics (including piecewise affine systems), piecewise polynomial controller dynamics, and piecewise polynomial protocols (characterized more precisely below) which include the well known Round Robin (RR) and Try-OnceDiscard (TOD) protocols, Lyapunov functions can be constructed using sum of squares (SOS) tools [19,30,31]. As a result, this will lead to LMI-based tests for stability given bounds on the delays and transmission intervals. As such, this approach has the following beneficial features: (1) we can deal with nonlinear (piecewise) polynomial controllers and systems, while the constructive conditions in the discrete-time and sampled-data approach only can handle linear plants and controllers; (2) we can easily incorporate non-zero lower bounds on the transmission interval and delays; (3) we allow more flexibility in the Lyapunov functions thereby obtaining less conservative results; (4) we can include performance analysis in terms of $\mathcal{L}_{p}$-gains of the continuoustime NCS, which is much harder for, for instance, the discrete-time approach due to the absence of intersample behavior in the discrete-time models; (5) we obtain an automated method to address stability analysis problems in NCS; (6) we do not have to discretize nor perform any polytopic overapproximations as in the discrete-time approach. Hence, the SOS-based stability analysis for NCSs offers many (different) advantages with respect to the various methods that already exist in the literature.

The rest of the paper is organized as follows. In Section 2 , we will present the general NCS model. Next in Section 3, we will provide the relevant theory for hybrid system stability and show how the stability conditions can be transformed into SOS problems. In particular, we present a procedure that can deal with the characteristics and peculiarities of the NCS models at hand. Finally in Section 4, four examples will demonstrate the strengths of the proposed approach. 


\section{NCS Description}

In this section, we describe a general NCS model discussed in [15], which extends earlier work [26], that was inspired by [37]. This model includes time-varying delays, time-varying transmission intervals and a shared communication medium. In addition, dropouts might be included by modeling them as prolongations of transmission intervals.

\subsection{Description of the NCS}

Consider the continuous-time plant

$$
\dot{x}_{p}=f_{p}\left(x_{p}, \hat{u}\right), \quad y=g_{p}\left(x_{p}\right)
$$

in which $x_{p} \in \mathbb{R}^{n_{p}}$ denotes the state of the plant, $\hat{u} \in \mathbb{R}^{n_{u}}$ denotes the control values being implemented at the plant and $y \in \mathbb{R}^{n_{y}}$ is the output of the plant. The plant is controlled over a shared communication network by a controller, given by

$$
\dot{x}_{c}=f_{c}\left(x_{c}, \hat{y}\right), \quad u=g_{c}\left(x_{c}, \hat{y}\right)
$$

where the variable $x_{c} \in \mathbb{R}^{n_{c}}$ is the state of the controller, $\hat{y} \in \mathbb{R}^{n_{y}}$ contains the most recent output measurements of the plant that are available at the controller and $u \in \mathbb{R}^{n_{u}}$ denotes the controller output. The presence of a communication network causes $u \neq \hat{u}$ and $y \neq \hat{y}$, as will be explained next. In particular, the considered NCS setup assumes that the sensor acts in a time-driven fashion and that both the controller and the actuator act in an event-driven fashion (i.e. responding instantaneously to newly arrived data). The controller, sensors, and actuators are connected through a shared network subject to varying transmission intervals and varying delays. The fact that the network imposes a shared communication medium implies that not all sensor and actuator data can be transmitted simultaneously and scheduling by a network protocol is necessary.

\subsubsection{Varying Transmission Intervals}

At the transmission instants, $t_{k} \in \mathbb{R}_{\geq 0}, k \in \mathbb{N}$, the plant outputs and control values are sampled and sent over the network. The transmission instants $t_{k}$ satisfy

$$
t_{k}=\sum_{i=0}^{k-1} h_{i} \quad \forall k \in \mathbb{N}
$$

which are non-equidistantly spaced in time due to the time-varying transmission intervals $h_{k}:=t_{k+1}-t_{k}$, with $h_{k} \in\left[h_{\min }, h_{\max }\right]$ for all $k \in \mathbb{N}$, for some $0 \leq h_{\min } \leq$ $h_{\max }$. In the literature, see e.g. [15, 26,37], where often $h_{\min }=0, h_{\max }$ is sometimes called the maximally allowable transmission interval (MATI). We assume that the sequence of transmission instants $t_{0}, t_{1}, t_{2}, \ldots$ is strictly increasing in the sense that $t_{k+1}>t_{k}$, for all $k \in \mathbb{N}$ and $\lim _{k \rightarrow \infty} t_{k}=\infty$.

\subsubsection{Varying Delays}

The transmitted control values and measurement values are received after a delay $\tau_{k} \in \mathbb{R}_{\geq 0}$, with $\tau_{k} \in\left[\tau_{\text {min }}, \tau_{\text {max }}\right]$, for all $k \in \mathbb{N}$ where $0 \leq \tau_{\text {min }} \leq \tau_{\text {max }}$. In the literature (e.g. [15], where $\tau_{\min }=0$ ), $\tau_{\max }$ is sometimes called the maximal allowable delay (MAD). The delay is primarily due to the speed at which the data travels through the network. Given the speed of current communication hardware, it is realistic in several practical situations to assume that the delay is smaller than the transmission interval required for control purposes. As such, the following standing assumption is adopted to describe the admissible range of transmission intervals and delays.

Assumption 1 The transmission intervals satisfy $0 \leq$ $h_{\text {min }} \leq h_{k} \leq h_{\max }$ and $h_{k}>0$ for all $k \in \mathbb{N}$ such that $\lim _{k \rightarrow \infty} t_{k}=\infty$, and the delays satisfy $0 \leq \tau_{\text {min }} \leq \tau_{k} \leq$ $\min \left\{\tau_{\max }, h_{k}\right\}, k \in \mathbb{N}$.

The latter condition implies that each transmitted packet arrives before the next sample is taken (i.e. the so-called small delay case). Hence, under Assumption 1 , without loss of generality we can assume that $\tau_{\max } \leq h_{\max }$

\subsubsection{Shared Communication Medium}

A shared communication medium prevents all control values and measurement values from being sent at each transmission time. A collection of sensors and/or actuators that are allowed to transmit their data simultaneously over the network is called a node. In practical situations each node typically corresponds to either a collection of sensors or a collection of actuators. At each transmission time $t_{k}, k \in \mathbb{N}$, a protocol determines which node $\sigma_{k} \in\{1,2, \ldots, N\}$ is granted access to the network. We will use the left-continuous piecewise constant function $\sigma: \mathbb{R}_{\geq 0} \rightarrow\{1, \ldots, N\}$ given by

$$
\sigma(t)=\sigma_{k}, t \in\left(t_{k}, t_{k+1}\right], k \in \mathbb{N}
$$

such that $\sigma(t), t \in \mathbb{R}_{\geq 0}$ is the node that was granted access at the most recent transmission instant before time $t$. The sensors/actuators corresponding to the node, which are granted access, collect their corresponding values in $y\left(t_{k}\right)$ or $u\left(t_{k}\right)$ that will be sent over the communication network. They will arrive after a transmission delay of $\tau_{k}$ time units, i.e. at time $t_{k}+\tau_{k}$.

To model this setup properly, we will denote the latest information available at the plant as $\hat{u}$ and the latest information available at the controller as $\hat{y}$. In addition, 
we will define the network-induced errors $e_{y}=\hat{y}-y$ and $e_{u}=\hat{u}-u$ to describe the difference between what is the most recent information that is available at the controller/plant and the current value of the plant/controller output, respectively. Sometimes we will write $e=\left(e_{u}, e_{y}\right) \in \mathbb{R}^{n_{e}}$, where we use the notation $\left(e_{y}, e_{u}\right):=\left[e_{y}^{\top}, e_{u}^{\top}\right]^{\top}$ and $n_{e}=n_{y}+n_{u}$. In between the updates of $\hat{y}$ and $\hat{u}$, the network is assumed to operate in a zero-order-hold $(\mathrm{ZOH})$ fashion, meaning that the values of $\hat{y}$ and $\hat{u}$ remain constant in between the updating times $t_{k}+\tau_{k}$ and $t_{k+1}+\tau_{k+1}$. At times $t_{k}+\tau_{k}$, $k \in \mathbb{N}$, the updates satisfy

$$
\begin{aligned}
& \hat{y}\left(\left(t_{k}+\tau_{k}\right)^{+}\right)=y\left(t_{k}\right)+h_{y}\left(\sigma\left(t_{k}\right), e\left(t_{k}\right)\right) \\
& \hat{u}\left(\left(t_{k}+\tau_{k}\right)^{+}\right)=u\left(t_{k}\right)+h_{u}\left(\sigma\left(t_{k}\right), e\left(t_{k}\right)\right) .
\end{aligned}
$$

The functions $h_{y}$ and $h_{u}$ are update functions that are related to the protocol, which will be explained in more detail in the next paragraph. We will combine $h_{u}$ and $h_{y}$ into one function $h$ as $h=\left(h_{u}, h_{y}\right)$. Based on (5) and the $\mathrm{ZOH}$ assumption, the network-induced error behaves at the update times $t_{k}+\tau_{k}$ according to

$$
e\left(\left(t_{k}+\tau_{k}\right)^{+}\right)=h\left(\sigma\left(t_{k}\right), e\left(t_{k}\right)\right)-e\left(t_{k}\right)+e\left(t_{k}+\tau_{k}\right) .
$$

See [15] and [26] for more details on (6) and the complete NCS model setup.

Remark 1 Note that here we adopt a ZOH assumption between updates, as it is the standard convention for digital-to-analog signal conversion. Alternatively, a generalized (possibly model-based) hold which predicts future control values can be implemented at the actuators and a model-based estimator can be implemented at the controller, as in e.g. [4, 32].

To provide insight into how the protocol $h$ operates, let us consider the two most well-known protocols being the Round Robin (RR) protocol and the Try-OnceDiscard (TOD) protocol. To introduce them, the error vector $e$ will be partitioned as $e=\left(e_{1}, e_{2}, \ldots, e_{N}\right)$ according to the nodes and the function $h$ will be partitioned similarly as $h=\left(h_{1}, \ldots, h_{N}\right)$, where $N$ is the number of nodes. In many situations, including the RR and TOD protocols, the protocol function $h$ is given as $h(\sigma, e)=\tilde{h}(\delta(\sigma, e), e)$, where $\tilde{h}(j, e)$ describes how the updates in (5) take place when node $j$ gets access to the network, and $\delta:\{1, \ldots, N\} \times \mathbb{R}^{n_{e}} \rightarrow\{1, \ldots, N\}$ describes which node is given access. In particular, which node $\sigma_{k} \in\{1, \ldots, N\}$ gets access to the network at the transmission time $t_{k}$ is based on the previous node $\sigma\left(t_{k}\right)$ that got access at $t_{k-1}$, and the network-induced error $e\left(t_{k}\right)$ at $t_{k}$. Although other choices for $\tilde{h}$ are possible (see e.g. Example 2 in [28]), in most cases $\tilde{h}$ is defined as

$$
\tilde{h}_{i}(j, e):=\left\{\begin{array}{l}
0, \text { if } i=j, \\
e_{i}, \text { otherwise }
\end{array}\right.
$$

for $i, j \in\{1,2, \ldots, N\}$ and $e \in \mathbb{R}^{n_{e}}$. Hence, $\tilde{h}$ expresses that when node $j$ gets network access, the outputs $y_{i}$ that belong to node $j$ satisfy $\hat{y}_{i}\left(\left(t_{k}+\tau_{k}\right)^{+}\right)=y_{i}\left(t_{k}\right)$, according to (5), while the outputs not corresponding to node $j$ are not updated. Similar statements hold, of course, for the control inputs $u$. This indicates that the values of $\hat{y}$ and $\hat{u}$ corresponding to the node that gets access are updated at time $t_{k}+\tau_{k}$ to the values of $y$ and $u$ at time $t_{k}$, which is natural for many practical cases. Using the definition of $\tilde{h}$ as in (7), the RR protocol is now specified by $h(\sigma, e)=\tilde{h}\left(\delta_{R R}(\sigma, e), e\right)$ with $\delta_{R R}(\sigma, e)$ defined for $\sigma \in\{1, \ldots, N\}$ and $e \in \mathbb{R}^{n_{e}}$ by

$$
\delta_{R R}(\sigma, e):=(\sigma \bmod N)+1
$$

where mod denotes the modulo operator. For the TOD protocol, $h(\sigma, e)=\tilde{h}\left(\delta_{T O D}(\sigma, e), e\right)$ with $\delta_{T O D}(\sigma, e)$ defined for $\sigma \in\{1, \ldots, N\}$ and $e \in \mathbb{R}^{n_{e}}$ by

$$
\delta_{T O D}(\sigma, e):=\arg \max \left|e_{j}\right|, \quad j=1, \ldots, N .
$$

where $|\cdot|$ denotes the Euclidian norm. Hence, the RR protocol grants each node access to the network in a periodic fashion, whereas the TOD protocol grants access to whichever node has the largest network-induced error $\left|e_{i}\left(t_{k}\right)\right|$ at transmission time $t_{k}, k \in \mathbb{N}$. In the case where two nodes have the largest error magnitude, one node is chosen arbitrarily.

The problem that we aim to solve in this paper is to determine stability of the NCS given the protocol, such as the RR or TOD protocol, and bounds $h_{\min }, h_{\max }, \tau_{\min }$ and $\tau_{\max }$ as in Assumption 1, or determine these bounds such that stability is guaranteed.

\subsection{Hybrid System Formulation}

To facilitate the stability analysis, the NCS model is transformed into a hybrid system [13], [15] of the form

$$
\begin{aligned}
\dot{\xi} & =F(\xi), \quad \xi \in C, \\
\xi^{+} & =G(\xi), \quad \xi \in D,
\end{aligned}
$$

where $C$ and $D$ are subsets of $\mathbb{R}^{n_{\xi}}, F: C \rightarrow \mathbb{R}^{n_{\xi}}$ and $G: D \rightarrow \mathbb{R}^{n_{\xi}}$ are mappings and $\xi^{+}$denotes the value of the state directly after the reset. We denote the hybrid system (10) for shortness sometimes by its data $(C, D, F, G)$.

To transform the NCS setup given by (1)-(2) and (5) into (10), the auxiliary variables ${ }^{2} s \in \mathbb{R}^{n_{e}}, \tau \in \mathbb{R}_{\geq 0}$,

\footnotetext{
2 Here we slightly divert from the models as used in $[15,26,37]$ where the hybrid system had the state $\kappa \in \mathbb{N}$, which indicated the number of the transmission, instead of $\sigma \in\{1, \ldots, N\}$. This modification is more convenient for our purposes of stability analysis based on sum of squares.
} 
$\sigma \in\{1, \ldots, N\}$ and $\ell \in\{0,1\}$ are introduced to reformulate the model in terms of so-called flow equations (10a) and reset equations (10b). The variable $s$ is an auxiliary variable containing the memory storing the value $h\left(\sigma\left(t_{k}\right), e\left(t_{k}\right)\right)-e\left(t_{k}\right)$ at $t_{k}$ for the update of $e$ at the update instant $t_{k}+\tau_{k}$ as in (6), $\tau$ is a timer to keep track of how much time elapsed since the last transmission event, $\sigma$ denotes the latest node that got access to the network, as in (4), and $\ell$ is a Boolean keeping track whether the next event is a transmission event or an update event. To be precise, when $\ell=0$ the next event will be related to transmission (at times $t_{k}, k \in \mathbb{N}$ ) and when $\ell=1$ the next event will be an update (at times $t_{k}+\tau_{k}, k \in \mathbb{N}$ ).

The state of our hybrid system $\Sigma_{N C S}$ is chosen as $\xi=(x, e, s, \tau, \sigma, \ell) \in \mathbb{R}^{n_{\xi}}$, where $x=\left(x_{p}, x_{c}\right)$. The continuous flow map $F$ can now be defined as

$$
F(\xi):=(f(x, e), g(x, e), 0,1,0,0),
$$

where $f, g$ are appropriately defined functions depending on $f_{p}, g_{p}, f_{c}$ and $g_{c}$. See [26] for the explicit expressions of $f$ and $g$. The state $s$ is specified as a memory variable, therefore, $\dot{s}=0$. The state $\tau$ is a timer variable and thus $\dot{\tau}=1$. Finally, the states $\sigma$ and $\ell$ are integer valued and leads to $\dot{\sigma}=\dot{\ell}=0$. Flow according to $\dot{\xi}=F(\xi)$ occurs when the state $\xi$ lies in the flow set

$$
\begin{array}{r}
C:=\left\{\xi \in \mathbb{R}^{n_{\xi}} \mid\left(\ell=0 \wedge \tau \in\left[0, h_{\max }\right]\right)\right. \\
\left.\vee\left(\ell=1 \wedge \tau \in\left[0, \tau_{\max }\right]\right)\right\},
\end{array}
$$

where $\wedge$ denotes the logical 'and' operator and $\vee$ denotes the logical (non-exclusive) 'or' operator. The jump map $G$ inducing resets

$$
\left(x^{+}, e^{+}, s^{+}, \tau^{+}, \sigma^{+}, \ell^{+}\right)=G(x, e, s, \tau, \sigma, \ell),
$$

is obtained by combining the "transmission reset relations," active at the transmission instants $\left\{t_{k}\right\}_{k \in \mathbb{N}}$, and the "update reset relations", active at the update instants $\left\{t_{k}+\tau_{k}\right\}_{k \in \mathbb{N}}$. Using (6), the jump map $G$ is defined at the transmission resets (when $\ell=0$ ) as

$$
G(x, e, s, \tau, \sigma, 0):=(x, e, h(\sigma, e)-e, 0, \delta(\sigma, e), 1)
$$

and the update resets (when $\ell=1$ ) as

$$
G(x, e, s, \tau, \sigma, 1):=(x, s+e, 0, \tau, \sigma, 0) .
$$

Note that when $\ell=1$ the update $s^{+}$can be chosen arbitrarily since the state variable $s$ does not influence the future evolution of other state variables. We choose $s^{+}:=0$ for convenience. The jump map $G$ is allowed to reset the system when the state is in the jump set

$$
\begin{array}{r}
D:=\left\{\xi \in \mathbb{R}^{n_{\xi}} \mid\left(\ell=0 \wedge \tau \in\left[h_{\min }, h_{\max }\right]\right)\right. \\
\left.\vee\left(\ell=1 \wedge \tau \in\left[\tau_{\min }, \tau_{\max }\right]\right)\right\} .
\end{array}
$$

Note that the sets $C$ and $D$ are defined by a combination of the state variables $\ell$ and $\tau$. Moreover, notice that in (13) and (14), $\ell$ changes its value from 0 to 1 and viceversa, respectively, and $\tau$ is reset to 0 only in (13). Due to this combination of $\ell$ and $\tau$ and Assumption 1, only one timer variable is needed for constraining both delays and transmission times.

Finally, we define the equilibrium set of the hybrid system

$$
\mathcal{A}:=\{\xi \in C \cup D \mid x=0 \wedge e=s=0\},
$$

for which we would like to prove stability. Hence, the informal stability problem phrased at the end of Section 2.1 translates now to the question of determining global asymptotic stability (GAS) of the set $\mathcal{A}$ for $\Sigma_{N C S}:=(C, D, F, G)$ (see [13] for exact definitions of global asymptotic stability of sets). For the remainder of the paper, we will define

$$
\chi:=(x, e, s) \in \mathbb{R}^{n_{\chi}},
$$

as this is the part of the state vector for which we would like to show convergence to zero.

Remark 2 The sampled-data system as considered in [22], which lumped the sensor-controller and controller-actuator delays into one delay, was modeled as an impulsive delay-differential equation and focused on linear plant dynamics $\dot{x}_{p}=A x_{p}+B \hat{u}$ with system matrix $A$, input matrix $B$ and state feedback controllers of the form $u=-K x_{p}$ (i.e. $y=x_{p}=x$ ). This model can also be expressed in this hybrid framework by omitting $\sigma, e_{u}$ and $x_{c}$ and taking $f(x, e)=(A-B K) x-B K e$, $g(x, e)=(-A+B K) x+B K e$ and $h(\sigma, e)=0$. Note that, $h(\sigma, e)=0$ implies that (5) simplifies to $\hat{y}\left(\left(t_{k}+\right.\right.$ $\left.\left.\tau_{k}\right)^{+}\right)=y\left(t_{k}\right)$.

\section{$3 \quad$ Stability Analysis}

In this section, we will show how the set $\mathcal{A}$ of the hybrid NCS model $\Sigma_{N C S}$ can be shown to be GAS by exploiting SOS techniques. We will first state some fundamental hybrid system stability results relevant to our purposes and then present the corresponding SOS theorems, which will be exploited to set up SOS-based stability conditions for the presented NCS model.

\subsection{Stability of Hybrid Systems}

First we will use the following definition to specify a Lyapunov function candidate $V: \operatorname{dom} V \rightarrow \mathbb{R}$, with dom $V \subseteq \mathbb{R}^{n_{\xi}}$, for a hybrid system as in (10). We will use the concept of a sublevel set of $V$ on a subset $\Xi$ of $\operatorname{dom} V$, which is a set of the form $\{\xi \in \Xi \mid V(\xi) \leq c\}$ for some $c \in \mathbb{R}$. 
Definition 1 [13] Consider a hybrid system $\Sigma=$ $(C, D, F, G)$ and a compact set $\mathcal{A} \subset \mathbb{R}^{n_{\xi}}$. The function $V: \operatorname{dom} V \rightarrow \mathbb{R}$, with $C \cup D \subseteq \operatorname{dom} V$, is a Lyapunov function candidate for $(\Sigma, \mathcal{A})$ if

(i.) $V$ is continuous and nonnegative on $(C \cup D) \backslash \mathcal{A} \subset$ dom $V$,

(ii.) $V$ is continuously differentiable on an open set $\mathcal{O}$ (iii.) satisfying $C \backslash \mathcal{A} \subset \mathcal{O} \subset$ dom $V$,

$$
\lim _{x \rightarrow \mathcal{A}, x \in \operatorname{dom} V \cap(C \cup D)} V(x)=0,
$$

(iv.) the sublevel sets of $V$ on $\operatorname{dom} V \cap(C \cup D)$ are compact.

To prove GAS of the set $\mathcal{A}$, we will make use of the following theorem, where we use the notation $\langle\cdot, \cdot\rangle$ to denote the standard inner product in Euclidean spaces.

Theorem 1 Consider a hybrid system $\Sigma=(C, F, D, G)$ and a compact set $\mathcal{A} \subset \mathbb{R}^{n_{\xi}}$ satisfying $G(D \cap \mathcal{A}) \subset \mathcal{A}$. If every solution of $\Sigma$ exists for all times $t \in[0, \infty)$ and there exists a Lyapunov function candidate $V$ for $(\Sigma, \mathcal{A})$ that satisfies Definition 1 and

$$
\begin{array}{r}
\langle\nabla V(\xi), F(\xi)\rangle<0 \text { for all } \xi \in C \backslash \mathcal{A} \\
V(G(\xi))-V(\xi) \leq 0 \text { for all } \xi \in D \backslash \mathcal{A},
\end{array}
$$

then the set $\mathcal{A}$ is $G A S$.

Proof The proof can be based on [13].

\subsection{Stability using SOS techniques}

Constructing suitable Lyapunov functions to prove stability is known to be a hard problem, certainly in the nonlinear and hybrid context. Here, we provide a computational approach to this problem based on polynomial Lyapunov functions and sum of squares (SOS) techniques $[5,17,19,29-31]$. To formally define a SOS we use the following definition, in which we use the notation $\mathbb{R}\left[x_{1}, \ldots, x_{n}\right]$ to denote the set of polynomials in $n$ variables $x_{1}, \ldots, x_{n}$ with real coefficients.

Definition 2 A polynomial $p \in \mathbb{R}\left[x_{1}, \ldots, x_{n}\right]$ is called a sum of squares if there exist $m \in \mathbb{N}$ and polynomials $p_{1}, p_{2}, \ldots, p_{m} \in \mathbb{R}\left[x_{1}, \ldots, x_{n}\right]$ such that $p(x)=\sum_{i=1}^{m} p_{i}^{2}(x)$ for all $x \in \mathbb{R}^{n}$.

Based on this definition, which implies that sum of squares are always nonnegative, inequalities, as in (18) and (19), can be guaranteed to be true if their left-hand sides can be expressed as sums of squares (where Sprocedure like relaxations [2] can be used to incorporate the regional information $\xi \in C \backslash \mathcal{A}$ in (18) and $\xi \in D \backslash \mathcal{A}$ in $(19))$. The appeal of SOS is that the solution can be computed using convex semidefinite programming techniques. Indeed $p(x)=\sum_{i=1}^{m} p_{i}^{2}(x)$ can be checked by finding a positive semidefinite matrix $Q$, and a vector of monomials $Z(x)$ such that $p(x)=Z^{\top}(x) Q Z(x)$ for all $x$. If a positive semidefinite matrix $Q$ is found, we can use Choleski factorization to obtain $Q=L^{\top} L$ for some matrix $L$ and thus write $p(x)$ as a sum of squares $p(x)=Z^{\top}(x) L^{\top} L Z(x)=|L Z(x)|^{2}=\sum_{i}\left(L_{i} Z(x)\right)^{2}$, where $L_{i}$ denotes the $i^{\text {th }}$ row of $L$, see e.g. $[5,19,31]$ for more details.

In the context of stability of hybrid systems (10), when $F$ and $G$ are piecewise polynomial functions (which in the case of the NCS models presented earlier, is true when $f_{c}, f_{p}, h$ are piecewise polynomial and $g_{c}, g_{p}$ are polynomial) on their domains $C$ and $D$, the Lyapunov stability conditions in Theorem 1 can be transformed into a set of polynomial inequalities. To formalize this idea, we provide the following two definitions.

Definition $3 A$ set $\mathcal{B}$ is called a basic semialgebraic set if it can be described as

$$
\begin{aligned}
\mathcal{B}=\left\{x \in \mathbb{R}^{n} \mid b_{j}(x) \geq 0, j=1, \ldots, n_{\mathcal{B}}\right. \text { and } & \\
& \left.\bar{b}_{l}(x)=0, l=1, \ldots, \bar{n}_{\mathcal{B}}\right\}
\end{aligned}
$$

for certain polynomials $b_{j} \in \mathbb{R}\left[x_{1}, \ldots, x_{n}\right], j=1, \ldots, n_{\mathcal{B}}$ and $\bar{b}_{l} \in \mathbb{R}\left[x_{1}, \ldots, x_{n}\right], l=1, \ldots, \bar{n}_{\mathcal{B}}$.

Definition 4 A function $p: \Omega \rightarrow \mathbb{R}$ with $\Omega \subseteq \mathbb{R}^{n}$ is called piecewise polynomial if there are $M$ basic semialgebraic sets $\Omega_{1}, \ldots, \Omega_{M}$ and polynomials $p_{1}, \ldots, p_{M} \in$ $\mathbb{R}\left[x_{1}, \ldots, x_{n}\right]$ such that

$$
\begin{aligned}
& \text { (i) } \Omega=\bigcup_{i=1}^{M} \Omega_{i}, \\
& \text { (ii) } \forall x \in \Omega \text { there exists an } i \in\{1, \ldots, M\} \\
& \text { such that } p(x)=p_{i}(x) \text { and } x \in \Omega_{i} .
\end{aligned}
$$

Remark 3 Note that in this definition the sets $\Omega_{1}, \ldots, \Omega_{N}$ may overlap, which is convenient as basic semialgebraic sets are closed and are not given by strict inequalities. Basically stability of the hybrid system will be proven no matter which $p_{i}(x)$ (e.g. in the flow or jumps of (10)) will be chosen in regions that overlap. As such, also the arbitrariness in the TOD protocol (i.e. when two nodes have the same largest networked induced error, see (9) and the discussion after (9)) can be incorporated.

To apply SOS techniques to the hybrid model (10), $F: C \rightarrow \mathbb{R}^{n_{\xi}}$ and $G: D \rightarrow \mathbb{R}^{n_{\xi}}$ need to be piecewise polynomial as in Definition 4. The sets $C$ and $D$ can then be expressed as $C=\cup_{i=1}^{I} C_{i}$ and $D=\cup_{m=1}^{M} D_{m}$ with $C_{i}, i=1, \ldots, I$, and $D_{m}, m=1, \ldots, M$, basic semialgebraic sets, meaning that 


$$
\begin{gathered}
C_{i}=\left\{\xi \in \mathbb{R}^{n_{\xi}} \mid c_{i, j}(\xi) \geq 0, \text { for } j=1, . ., n_{C}^{i},\right. \\
\left.\bar{c}_{i, l}(\xi)=0, \text { for } l=1, . ., \bar{n}_{C}^{i}\right\}, \\
D_{m}=\left\{\xi \in \mathbb{R}^{n_{\xi}} \mid d_{m, j}(\xi) \geq 0, \text { for } j=1, . ., n_{D}^{m},\right. \\
\left.\bar{d}_{m, l}(\xi)=0, \text { for } l=1, . ., \bar{n}_{D}^{m}\right\}
\end{gathered}
$$

where $c_{i, j}, \bar{c}_{i, l}, d_{m, j}$ and $\bar{d}_{m, l} \in \mathbb{R}[\xi]$ are polynomials. Moreover, there are polynomials $F_{1}, \ldots, F_{I}$ and $G_{1}, \ldots, G_{M}$ such that for each $\xi \in C$ there is an $i \in\{1, \ldots, I\}$ such that $F(\xi)=F_{i}(\xi)$ and $\xi \in C_{i}$, and for each $\xi \in D$ there is an $m \in\{1, \ldots, M\}$ such that $G(\xi)=G_{m}(\xi)$ and $\xi \in D_{m}$. Hence, the hybrid system (10) can then also be expressed as

$$
\begin{aligned}
\dot{\xi} & =F_{i}(\xi), \quad \xi \in C_{i}, i=1, \ldots, I, \\
\xi^{+} & =G_{m}(\xi), \quad \xi \in D_{m}, m=1, \ldots, M
\end{aligned}
$$

Note that (22) allows for a slightly richer set of solutions than (10), see Remark 3. We will use the above notation to expand Theorem 1 in the spirit of [30] by applying a technique similar to the S-procedure [2], called the positivstellensatz $[19,31]$, in order to encode the information that the inequalities (18) and (19) only have to be satisfied on the sets $C \backslash \mathcal{A}$ and $D \backslash \mathcal{A}$ into the inequalities.

Theorem 2 Consider a hybrid system $\Sigma=(C, F, D, G)$ as in (22) with the sets $C=\cup_{i=1}^{I} C_{i}$ and $D=\cup_{m=1}^{M} D_{m}$, where $C_{i}$ is of the form (20) and $D_{m}$ is of the form (21), and $F_{i}$ and $G_{m}$ are polynomial functions for all $i=1, \ldots, I$ and $m=1, \ldots, M$. Furthermore, consider $a$ compact set $\mathcal{A} \subset \mathbb{R}^{n_{\xi}}$ satisfying $G(D \cap \mathcal{A}) \subset \mathcal{A}$. If every solution of $\Sigma$ exists for all times $t \in[0, \infty)$ and there exist (i.) a Lyapunov function candidate $V$ for $(\Sigma, \mathcal{A})$ that satisfies Definition 1, (ii.) polynomials $\bar{r}_{i, l}, \bar{s}_{m, l} \in \mathbb{R}[\xi]$ and (iii.) SOS polynomials $r_{i, j}, s_{m, j} \in \mathbb{R}[\xi]$ such that

$$
\begin{aligned}
& \left\langle\nabla V(\xi), F_{i}(\xi)\right\rangle+\sum_{j=1}^{n_{C}^{i}} r_{i, j}(\xi) c_{i, j}(\xi)+ \\
& +\sum_{l=1}^{\bar{n}_{C}^{i}} \bar{r}_{i, l}(\xi) \bar{c}_{i, l}(\xi)<0 \quad \forall \xi \notin \mathcal{A}, i=1, \ldots, I, \\
& V\left(G_{m}(\xi)\right)-V(\xi)+\sum_{j=1}^{n_{D}^{m}} s_{m, j}(\xi) d_{m, j}(\xi)+ \\
& \quad+\sum_{l=1}^{\bar{n}_{D}^{m}} \bar{s}_{m, l}(\xi) \bar{d}_{m, l}(\xi) \leq 0 \quad \forall \xi \notin \mathcal{A}, m=1, \ldots M,
\end{aligned}
$$

then the set $\mathcal{A}$ is $G A S$.

Proof If (23) holds, then since the terms $r_{i, j}(\xi) c_{i, j}(\xi)$ are nonnegative and the terms $\bar{r}_{i, j}(\xi) \bar{c}_{i, j}(\xi)$ are zero when $\xi \in C_{i}$, it must be true that $\left\langle\nabla V(\xi), F_{i}(\xi)\right\rangle<0$ when $\xi \in C_{i} \backslash \mathcal{A}$. Using the fact that $C=\cup_{i=1}^{I} C_{i}$ and for all $\xi \in C$ there exists an $i$ such that $\xi \in C_{i}$ and $F(\xi)=F_{i}(\xi)$, we have that $\langle\nabla V(\xi), F(\xi)\rangle<0$ when $\xi \in C \backslash \mathcal{A}$. Hence, (18) holds. By the same reasoning, if (24) holds then (19) must hold. Thus, if the function $V$ satisfies the hypotheses of Theorem 2 , then it also satisfies the hypotheses of Theorem 1 thereby proving GAS of $\mathcal{A}$.

Remark 4 The SOS relaxation technique as in Theorem 2 can also be applied to encode that the (polynomial) function $V$ only has to be nonnegative on $(C \cup D) \backslash \mathcal{A}$ (as required in Definition 1) into (polynomial) inequalities. We encode this by introducing polynomials $\bar{p}_{m, l}, \bar{q}_{i, l} \in$ $\mathbb{R}[\xi]$ and $S O S$ polynomials $p_{m, j}, q_{i, j} \in \mathbb{R}[\xi]$ leading to the following inequalities

$$
\begin{array}{r}
V(\xi)-\sum_{j=1}^{n_{C}^{i}} q_{i, j}(\xi) c_{i, j}(\xi)-\sum_{l=1}^{\bar{n}_{C}^{i}} \bar{q}_{i, l}(\xi) \bar{c}_{i, l}(\xi) \geq 0, \\
\forall \xi \notin \mathcal{A}, \forall i=1, \ldots, I, \\
V(\xi)-\sum_{j=1}^{n_{D}^{m}} p_{m, j}(\xi) d_{m, j}(\xi)-\sum_{l=1}^{\bar{n}_{D}^{m}} \bar{p}_{m, l}(\xi) \bar{d}_{m, l}(\xi) \geq 0, \\
\forall \xi \notin \mathcal{A}, \forall m=1, \ldots, M,
\end{array}
$$

which indeed guarantees that $V(\xi) \geq 0$ for $\xi \in(C \cup D) \backslash \mathcal{A}$.

Remark 5 In Theorem 2 and Remark 4 we only used the polynomial constraints that define $C_{i}$ and $D_{m}$ themselves to relax the stability conditions, and not their products. For instance, in (23) we could have also added terms as $\sum_{l} \sum_{\tilde{l}} r_{i, l, \tilde{l}}(\xi) c_{i, l}(\xi) c_{i, \tilde{l}}(\xi)$ with $r_{i, l, \tilde{l}}(\xi) S O S$ and still guarantee that $\left\langle\nabla V(\xi), F_{i}(\xi)\right\rangle<0$ when $\xi \in C_{i} \backslash \mathcal{A}$. Clearly this would result in a further relaxation with respect to (23) (at the expense of a larger SOS program). See [5, 19,31] for more details on these relaxations, which can be implemented for the stability analysis of NCSs along the same lines as discussed in this paper. For ease of exposition we restricted ourselves to the form in (23), (24), (25) and (26) excluding such products.

SOS conditions only guarantee non-negativity of polynomials (i.e. non-strict inequalities) but proving asymptotic stability requires the Lyapunov derivative (23) being negative definite (satisfying a strict inequality). Thus, we need a way to verify that a given polynomial function is negative or positive definite by checking SOS (positive semidefinite) conditions. We will use the following proposition from [29] to check for positive definiteness of a given polynomial.

Proposition 1 Given a polynomial $p \in \mathbb{R}[\xi]$ of degree 
$2 d$, let $W(\xi)=\sum_{i=1}^{n_{\xi}} \sum_{j=1}^{d} \epsilon_{i, j} \xi_{i}^{2 j}$ be such that

$$
\sum_{j=1}^{d} \epsilon_{i, j}>\gamma \quad \text { for all } i=1, \ldots, n
$$

with $\gamma$ a positive number, and $\epsilon_{i, j} \geq 0$ for all $i$ and $j$. Then the condition

$$
p(\xi)-W(\xi) \geq 0 \quad(p(\xi)-W(\xi) \text { is } S O S)
$$

guarantees the positive definiteness of $p$, i.e. $p(\xi)>0$ for all $\xi \neq 0$.

Proposition 1 and Theorem 2 form the basis to build the SOS programs that can prove stability of our NCS model (10) with (11)-(15).

\subsection{Stability of Hybrid NCS models via SOS techniques}

In this section we will specify how to verify GAS of the set $\mathcal{A}$, as defined in (16), of the hybrid NCS models using SOS techniques. We will distinguish three cases. In all three cases, we choose a Lyapunov function candidate satisfying Definition 1. Specifically, the candidate we choose is polynomial and we impose as a constraint that it can be written as a SOS (using S-procedure like relaxations as in Remark 4) so that (i.) and (ii.) are satisfied. Condition (iii.) will be guaranteed by the explicit form and the continuity of the Lyapunov function candidate. Condition (iv.) will be satisfied by imposing that $V(\xi) \geq W(\chi)$ for all $\xi \in C \cup D \backslash \mathcal{A}$ where $\chi$ is defined in (17) and $W(\chi)$ is a (radially unbounded) function as given in Proposition 1. In this way, the conditions of Definition 1 are immediately satisfied.

Remark 6 In the case when $f$ and $g$ in (11) are linear functions, we do not need to satisfy Condition (iv.) of Definition 1 to guarantee GAS of the set $\mathcal{A}$. Removing Condition (iv.) implies that the Lyapunov function candidate can only prove local asymptotic stability in Theorem 1, see [13]. However, if $f$ and $g$ are linear functions and the protocol function $h$ satisfies $h(\sigma, \alpha e)=\alpha h(\sigma, e)$ for all $\sigma \in\{1, \ldots, N\}, e \in \mathbb{R}^{n_{e}}$ and $\alpha \geq 0$ (as is the case for the RR and TOD protocols), local asymptotic stability implies GAS due to the fact that an initial state $\left(x_{0}, e_{0}, s_{0}, \sigma_{0}, \ell_{0}, \tau_{0}\right)$ at $t=0$ has a solution $(x, e, s, \sigma, \ell, \tau)$ if and only if initial state $\left(\alpha x_{0}, \alpha e_{0}, \alpha s_{0}, \sigma_{0}, \ell_{0}, \tau_{0}\right)$ has a solation $(\alpha x, \alpha e, \alpha s, \sigma, \ell, \tau)$ for $\alpha>0$. Due to this positive homogeneity property, local asymptotic stability of $\mathcal{A}$ implies $G A S$ of $\mathcal{A}$.

In the remainder of this section we show how the SOS program can be set up to solve the NCS stability problem in three cases. First we will address the simplest case of a NCS without a shared communication medium, i.e. all sensor and controller data is transmitted at every transmission time as discussed briefly in Remark 2 . Then we will expand those results to solve the case with RR protocols and then finally consider TOD protocols. In each of these cases, the essential steps are the transformation of these three NCS variants into hybrid models (10) with $F: C \rightarrow \mathbb{R}^{n_{\xi}}$ and $G: D \rightarrow \mathbb{R}^{n_{\xi}}$ being piecewise polynomial as in Definition 4 leading to (22), and applying Theorem 2 together with Remark 4 and Proposition 1 to derive suitable SOS programs. For ease of exposition we will assume that $f$ and $g$ are polynomials. The extension to piecewise polynomials is straightforward. Also the extension to other protocols for which $\delta:\{1, \ldots, N\} \times \mathbb{R}^{n_{e}} \rightarrow\{1, \ldots, N\}$ is a piecewise constant function where the sets $S_{\sigma, j}=\left\{e \in \mathbb{R}^{n_{e}} \mid \delta(\sigma, e)=j\right\}$ are a finite union of basic semi-algebraic sets for each $\sigma, j \in\{1, \ldots, N\}$ can be obtained by following the same line of reasoning as below. In fact, in line with Definition 4 , we call the protocols with the above mentioned property piecewise polynomial protocols.

\subsubsection{Unshared Communication}

Without the communication medium being shared, it holds that $h(\sigma, e)=0$ for all $\sigma$ and $e$, and consequently, we can simplify the hybrid model by omitting $\sigma$ from the state $\xi$, resulting in $\xi=(\chi, \tau, \ell)$. Given the definitions of $C$ and $D$ for $\Sigma_{N C S}$, it is necessary to partition $C$ and $D$ based on the discrete state $\ell \in\{0,1\}$ as

$$
\begin{aligned}
& C_{0}=\left\{\xi \in \mathbb{R}^{n_{\xi}} \mid \ell=0, \tau \geq 0, h_{\max }-\tau \geq 0\right\} \\
& C_{1}=\left\{\xi \in \mathbb{R}^{n_{\xi}} \mid \ell=1, \tau \geq 0, \tau_{\max }-\tau \geq 0\right\}
\end{aligned}
$$

with the corresponding polynomial flow map

$$
\begin{aligned}
& F_{0}(\xi)=F_{1}(\xi)=F(\chi, \tau, \ell)= \\
& (f(x, e), g(x, e), 0,1,0)
\end{aligned}
$$

and

$$
\begin{aligned}
& D_{0}=\left\{\xi \in \mathbb{R}^{n_{\xi}} \mid \ell=0, \tau-h_{\min } \geq 0,\right. \\
& \left.h_{\max }-\tau \geq 0\right\}, \\
& D_{1}=\left\{\xi \in \mathbb{R}^{n_{\xi}} \mid \ell=1, \tau-\tau_{\min } \geq 0,\right. \\
& \left.\tau_{\max }-\tau \geq 0\right\},
\end{aligned}
$$

with the corresponding polynomial jump maps

$$
\begin{aligned}
& G_{0}(\xi)=G_{0}(\chi, \tau, \ell)=(x, e,-e, 0,1), \\
& G_{1}(\xi)=G_{1}(\chi, \tau, \ell)=(x, s+e, 0, \tau, 0) .
\end{aligned}
$$

Note that $C=C_{0} \cup C_{1}$, with $C_{i}, i=0,1$, basic semialgebraic sets, satisfying (20) and $D=D_{0} \cup D_{1}$, with $D_{m}, m=0,1$, basic semialgebraic sets, satisfying $(21)$. In addition, the mappings $G_{0}, G_{1}$ and $F_{0}=F_{1}=F$ are polynomial functions, provided that $f$ and $g$ are polynomial functions. This shows that $F: C \rightarrow \mathbb{R}^{n_{\xi}}$ and $G: D \rightarrow \mathbb{R}^{n_{\xi}}$ are piecewise polynomial, under the standing assumption that $f$ and $g$ are polynomial. Using the 
above expressions for $C_{i}, i=0,1$ and $D_{m}, m=0,1$, the polynomials $c_{i, j}$ and $d_{m, j}$ are defined as shown in Table 1 .

\begin{tabular}{ll}
\hline$c_{i, j}$ & $d_{m, j}$ \\
\hline$c_{0,1}=\tau$ & $d_{0,1}=\tau-h_{\min }$ \\
$c_{0,2}=h_{\max }-\tau$ & $d_{0,2}=h_{\max }-\tau$ \\
$c_{1,1}=\tau$ & $d_{1,1}=\tau-\tau_{\min }$ \\
$c_{1,2}=\tau_{\max }-\tau$ & $d_{1,2}=\tau_{\max }-\tau$ \\
\hline
\end{tabular}

Table 1

SOS relaxations for a NCS with unshared communication.

We did not include the equality constraints (e.g. $\ell=0$ for $C_{0}$ or $\ell=1$ for $C_{1}$ ) in Table 1 as we will encode them through the use of multiple Lyapunov functions explicitly depending on $\ell$. The Lyapunov function candidate we propose to use is of the form ${ }^{3}$

$$
V(\xi)=V_{\ell}(\chi, \tau)=\varphi_{\ell}(\tau) \tilde{W}_{\ell}(\chi)
$$

We specify that the function $\varphi_{\ell} \in \mathbb{R}[\tau]$ is a polynomial of any degree and $\tilde{W}_{\ell} \in \mathbb{R}[\chi]$ is a polynomial with an even degree, $\ell=0,1$. This choice of Lyapunov function is inspired by $[3,15]$. To guarantee Condition $(i$.) of Definition 1, we will use the regional information $c_{i, j}$ (as in Theorem 2) to explicitly specify that $V_{\ell}(\xi)$ is nonnegative only when $\xi \in C \cup D=C$. Choosing $\tilde{W}_{\ell}$ such that $\tilde{W}_{\ell}(\chi)=0$ when $\chi=0$ ensures that Condition (iii.) of Definition 1 is satisfied. Combining Proposition 1 and Theorem 2 together with Remark 4 leads to the polynomial constraints as shown in Table 2, where the inequalities will be implemented through SOS conditions.

\begin{tabular}{c|l}
\hline \multicolumn{2}{c}{ Constraint Set - Unshared Communication } \\
\hline $1 \mathrm{a}$ & $\sum_{j=1}^{d} \epsilon_{\ell, i, j} \geq \gamma_{1}, \quad \epsilon_{\ell, i, j} \geq 0$ \\
$1 \mathrm{~b}$ & $\sum_{j=1}^{d} \eta_{\ell, i, j} \geq \gamma_{2}, \quad \eta_{\ell, i, j} \geq 0$ \\
2 & $V_{\ell}(\chi, \tau)-W_{1, \ell}(\chi)-\sum_{j=1}^{m_{C}} q_{\ell, j}(\chi, \tau) c_{\ell, j}(\tau) \geq 0$ \\
3 & $-\left\langle\nabla V_{\ell}(\chi, \tau), F(\xi)\right\rangle-W_{2, \ell}(\chi)-$ \\
& $\sum_{j=1}^{2} r_{\ell, j}(\chi, \tau) c_{\ell, j}(\tau) \geq 0$ \\
$4 \mathrm{a}$ & $V_{0}(\chi, \tau)-V_{1}\left(\bar{G}_{0}(\chi, \tau)\right)-$ \\
$4 \mathrm{~b}$ & $V_{1}(\chi, \tau)-V_{0}\left(\bar{G}_{1}(\chi, \tau)\right)-$ \\
& $\sum_{j=1}^{2} s_{0, j}(\chi, \tau) d_{0, j}(\tau) \geq 0$ \\
Table 2 & $q_{\ell, j}^{2}(\chi, \tau) \geq 0, r_{\ell, j}(\chi, \tau) \geq 0, s_{\ell, j}(\chi, \tau) \geq 0$ \\
\hline
\end{tabular}

SOS program for a NCS with unshared communication.

\footnotetext{
3 Note that the multiple Lyapunov function $V(\xi)=V_{\ell}(\chi, \tau)$ can be written as one single polynomial Lyapunov function $V(\xi)=\ell V_{1}(\chi, \tau)+(1-\ell) V_{0}(\chi, \tau)$.
}

In Table 2 , we use the notation $\bar{G}_{i}, i=0,1$ to denote the jump map $G_{i}, i=0,1$, restricted to the elements corresponding to $\chi$ and $\tau$, i.e.

$$
\begin{aligned}
& \bar{G}_{0}(\chi, \tau)=(x, e,-e, 0), \\
& \bar{G}_{1}(\chi, \tau)=(x, s+e, 0, \tau) .
\end{aligned}
$$

The constraints in Table 2 must hold for all $\ell \in\{0,1\}$ and $i \in\left\{1,2, . ., n_{\chi}\right\}$ and fixed $\gamma_{1}>0$ and $\gamma_{2}>0$. The functions $W_{1, \ell}, W_{2, \ell} \in \mathbb{R}[\chi]$ for $\ell=0,1$ are defined as

$$
\begin{aligned}
& W_{1, \ell}(\chi)=\sum_{i=1}^{n_{\chi}} \sum_{j=1}^{d} \epsilon_{\ell, i, j} \chi_{i}^{2 j} \\
& W_{2, \ell}(\chi)=\sum_{i=1}^{n_{\chi}} \sum_{j=1}^{d} \eta_{\ell, i, j} \chi_{i}^{2 j}
\end{aligned}
$$

as in Proposition 1. These functions only need to depend on $\chi=(x, e, s)$ to guarantee (23) of Theorem 2 and (iv.) of Definition 1 because $\mathcal{A}=\{\xi \in C \cup D \mid \chi=0\}$. Note that we did not need to apply (26) of Remark 4 since $D \subseteq C$. Constraint 3 in Table 2 is derived from combining (28) and (23).

Remark 7 Besides choosing the orders of $\varphi_{\ell}$ and $\tilde{W}_{\ell}$ in the Lyapunov function itself, the design freedom left available is to choose the order of the polynomials $q_{\ell, j}(\chi, \tau), r_{\ell, j}(\chi, \tau)$ and $s_{\ell, j}(\chi, \tau)$ such that the order of the product $q_{\ell, j}(\chi, \tau) c_{\ell, j}(\tau)$ matches the order of $V_{\ell}(\chi, \tau)$, the order of $r_{\ell, j}(\chi, \tau) c_{\ell, j}(\tau)$ matches the order of $\left\langle\nabla V_{\ell}(\chi, \tau), F(\xi)\right\rangle$ and the order of $s_{\ell, j}(\chi, \tau) d_{\ell, j}(\tau)$ matches the order of $V_{\ell}(\chi, \tau)$. The motivation behind this suggestion is to create sufficient freedom for the relaxation terms in Table 2 to be effective but do not become computationally burdensome.

Hence, based on the previous reasoning, the feasibility of the SOS constraints in Table 2 guarantee GAS of a NCS with varying delays and varying transmission intervals, but without a shared communication medium. The communication medium being shared will be considered in the following two sections for the RR and TOD protocols. Other piecewise polynomial protocols can be handled in a similar fashion.

\subsubsection{Round Robin Protocol}

In the case of the Round Robin (RR) protocol, we exploit the periodicity present in the RR protocol $G_{0}(\chi, \tau, \sigma, \ell)=G_{0}(\chi, \tau, \sigma+N, \ell)$ to modify the region $D_{0}$ to be node-dependent in the sense that the jump map $G_{0}$ can be written in a piecewise polynomial form. Specifically, we partition $D_{m}, m=0,1$, as in (31), 
further as

$$
\begin{aligned}
& D_{0,1}=\left\{\xi \in \mathbb{R}^{n_{\xi}} \mid \ell=0, \tau-h_{\min } \geq 0\right. \\
& \left.h_{\max }-\tau \geq 0, \sigma=N\right\} \\
& D_{0, j}=\left\{\xi \in \mathbb{R}^{n_{\xi}} \mid \ell=0, \tau-h_{\min } \geq 0\right. \\
& \left.\quad h_{\max }-\tau \geq 0, \sigma+1=j\right\} \\
& D_{1}=\left\{\xi \in \mathbb{R}^{n_{\xi}} \mid \ell=1, \tau-\tau_{\min } \geq 0, \tau_{\max }-\tau \geq 0\right\}
\end{aligned}
$$

for $j=2, \ldots, N$. Now $G$ is specified by

$$
\begin{aligned}
G_{0, j}(\chi, \tau, \sigma, \ell) & =(x, e, \tilde{h}(j, e)-e, 0, j, 1) \\
G_{1}(\chi, \tau, \sigma, \ell) & =(x, s+e, 0, \tau, \sigma, 0)
\end{aligned}
$$

for $j=1, \ldots, N$, where $\tilde{h}$ is given as in (7). Hence, $D_{0}=\cup_{j=1}^{N} D_{0, j}$ and each function $G_{0, j}$ is polynomial, thus we have obtained a hybrid system of the form (22). The definitions of $D_{0, j}, j=1, \ldots, N$, and $D_{1}$ allow us to again use the polynomials defined in Table 1.

The Lyapunov function we consider to prove stability for $\Sigma_{N C S}$ with RR protocols is the following:

$$
V(\xi)=V_{\ell, \sigma}(\chi, \tau)=\varphi_{\ell, \sigma}(\tau) \tilde{W}_{\ell, \sigma}(\chi) .
$$

The explicit dependence on $\sigma$ provides extra freedom in the Lyapunov function candidate. With this new Lyapunov candidate, we obtain, in a similar way as in Section 3.3.1, the constraint set as shown in Table 3. We use the notation $\bar{G}_{0, j}$ for $j=1, \ldots, N$ to denote the jump map $G_{0, j}$ restricted to the first elements corresponding to $\chi$ and $\tau$, i.e.,

$$
\bar{G}_{0, j}(\chi, \tau)=(x, e, \tilde{h}(j, e)-e, 0) .
$$

The constraints in Table 3 must hold for all $\ell \in$ $\{0,1\}, i \in\left\{1,2, . ., n_{\chi}\right\}, \sigma_{1} \in\{1,2, \ldots, N\}$ and $\sigma_{2} \in$ $\{1,2, \ldots, N-1\}$ and fixed $\gamma_{1}>0$ and $\gamma_{2}>0$. The functions $W_{1, \ell, \sigma_{1}}, W_{2, \ell, \sigma_{1}} \in \mathbb{R}[\chi]$ are of the form (34).

\subsubsection{Try-Once-Discard Protocol}

For the TOD protocol, we know that at the time a jump occurs when $\ell=0$, the node that is granted network access has a greater (or equal) error $\left|e_{j}\right|$ than all other

\begin{tabular}{|c|c|}
\hline \multicolumn{2}{|r|}{ Constraint Set - RR Protocol } \\
\hline $1 \mathrm{a}$ & $\sum_{j=1}^{d} \epsilon_{\ell, \sigma_{1}, i, j} \geq \gamma_{1}, \quad \epsilon_{\ell, \sigma_{1}, i, j} \geq 0$ \\
\hline $1 b$ & $\sum_{j=1}^{d} \eta_{\ell, \sigma_{1}, i, j} \geq \gamma_{2}, \quad \eta_{\ell, i, j} \geq 0$ \\
\hline 2 & $V_{\ell, \sigma_{1}}(\chi, \tau)-W_{1, \ell, \sigma_{1}}(\chi)-\sum_{j=1} q_{\ell, j}(\chi, \tau) c_{\ell, j}(\tau) \geq 0$ \\
\hline 3 & $-\left\langle\nabla V_{\ell, \sigma_{1}}(\chi, \tau), F(\xi)\right\rangle-W_{2, \ell, \sigma_{1}}(\chi)-$ \\
\hline \multirow{3}{*}{$4 \mathrm{a}$} & $\sum_{j=1}^{2} r_{\ell, j}(\chi, \tau) c_{\ell, j}(\tau) \geq 0$ \\
\hline & $V_{0, N}(\chi, \tau)-V_{1,1}\left(\bar{G}_{0,1}(\chi, \tau)\right)-$ \\
\hline & $\sum_{j=1}^{2} s_{0, j}(\chi, \tau) d_{0, j}(\tau) \geq 0$ \\
\hline \multirow[t]{2}{*}{$4 b$} & $V_{0, \sigma_{2}}(\chi, \tau)-V_{1, \sigma_{2}+1}\left(\bar{G}_{0, \sigma_{2}+1}(\chi, \tau)\right)-$ \\
\hline & $\sum_{j=1}^{2} s_{0, j}(\chi, \tau) d_{0, j}(\tau) \geq 0$ \\
\hline \multirow[t]{2}{*}{$4 c$} & $V_{1, \sigma_{1}}(\chi, \tau)-V_{0, \sigma_{1}}\left(\bar{G}_{1}(\chi, \tau)\right)-$ \\
\hline & $\sum_{j=1}^{2} s_{1, j}(\chi, \tau) d_{1, j}(\tau) \geq 0$ \\
\hline 5 & $q_{\ell, j}(\chi, \tau) \geq 0, r_{\ell, j}(\chi, \tau) \geq 0, s_{\ell, j}(\chi, \tau) \geq 0$ \\
\hline
\end{tabular}
nodes, i.e. $\left|e_{j}\right| \geq\left|e_{i}\right|$ for all $i$ (see (9)). We will use this knowledge to modify the region $D_{0}$ so that the jump map $G_{0}$ is written as a piecewise polynomial function. We express $D_{0}$ as $\cup_{j} D_{0, j}$ with $D_{0, j}, j=1, \ldots, N$, basic semialgebraic sets given by

$$
\begin{aligned}
D_{0, j}= & \left\{\xi \in \mathbb{R}^{n_{\xi}} \mid \ell=0, \tau-h_{\min } \geq 0,\right. \\
& \left.h_{\max }-\tau \geq 0,\left|e_{j}\right|^{2}-\left|e_{i}\right|^{2} \geq 0 \forall i=1, \ldots, N\right\} .
\end{aligned}
$$

In addition,

$$
D_{1}=\left\{\xi \in \mathbb{R}^{n_{\xi}} \mid \ell=1, \tau-\tau_{\min } \geq 0, \tau_{\max }-\tau \geq 0\right\} .
$$

The corresponding jump maps $G_{0, j}$ and $G_{1}$ are given by (35), the flow map $F_{i}$ is again described by (30) and the corresponding flow set $C_{i}, i=0,1$, is described by (29). Now we have arrived at a system of the form (22) and we can apply Theorem 2 together with Remark 4 and Proposition 1.

We consider the Lyapunov function (36) to prove stability for $\Sigma_{N C S}$ with TOD protocols. Notice that we do not need $\sigma$ in the hybrid model of the NCS as $h(\sigma, e)=h(e)$. However, we do use $\sigma$ to provide extra freedom in the (multiple) Lyapunov function (as in the RR case). The resulting constraint set guaranteeing GAS of $\mathcal{A}$ is shown in Table 4. The constraints must hold for all $\ell \in\{0,1\}$, $i \in\left\{1,2, . ., n_{\chi}\right\}$, and $\sigma_{1}, \sigma_{2} \in\{1,2, \ldots, N\}$ and fixed $\gamma_{1}>0$ and $\gamma_{2}>0$. The functions $W_{1, \ell, \sigma_{1}}, W_{2, \ell, \sigma_{1}} \in \mathbb{R}[\chi]$ are of the form (34).

\section{Comparative Examples}

We will illustrate our SOS approach on four different NCS examples. In the first example, we use the sampleddata model without the communication medium being shared. In the second and third example we study a linear plant connected to a linear controller via a shared network, and thus include network protocols. We compare the SOS approach developed in this paper with the technique developed in [15]. We show that our results are less conservative than the results obtained using the technique developed in [15]. As these three examples consider linear plants and controllers, the fourth example will illustrate that the SOS approach can easily be used to show stability for plants and controllers with 


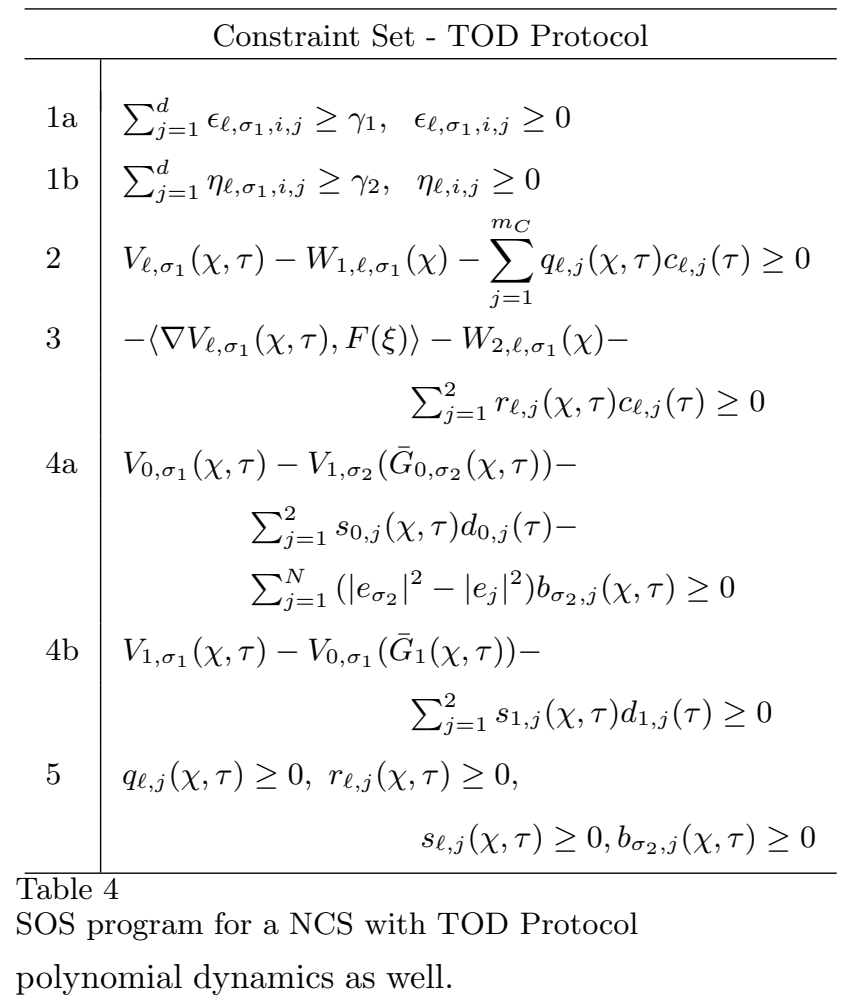

Our calculations result in tradeoff curves for combinations of $h_{\max }$ and $\tau_{\max }$ (where we take $h_{\min }=\tau_{\min }=0$ to be able to compare the results to [15]). These tradeoff curves are created by a gridding procedure in the sense that for different combinations of $h_{\max }$ and $\tau_{\max }$, the feasibility of the SOS program is assessed using SOSTOOLS [33].

\subsection{Example 1 - Unshared Communication}

A 'classic' and well studied system (see [20] and the reference therein), is given by (1), (2) where $\dot{x}_{p}=\hat{u}$, $y=x_{p}, u=-\hat{y}$. For constant transmission interval and no delays, the system can be guaranteed to be stable for transmission intervals up to 2 seconds. In [20], stability of the system for variable transmission intervals is guaranteed for $h_{k} \in\left[\begin{array}{ll}0 & 1.99\end{array}\right], k \in \mathbb{N}$ in a delay-free situation, which corresponds to $h_{\min }=0$ and a MATI $h_{\max }$ of 1.99 seconds. This does not include much conservatism, as can be concluded from the constant transmission interval result. The results obtained in [20], when delays are present, are given in Fig. 1.

Two SOS programs (SOSPs) are constructed with the constraints from Section 3.3.1. The first program used a third order $V_{\ell}$, which consisted of a linear function $\varphi_{\ell} \in \mathbb{R}[\tau]$ and quadratic $\tilde{W}_{\ell} \in \mathbb{R}[\chi]$, whereas the second program used a fifth order $V_{\ell}$, which consisted of a third order function for $\varphi_{\ell}$ and quadratic $\tilde{W}_{\ell}$. Already with $\varphi_{\ell}$ being a polynomial of third order, the results of [20], which according to the above do not contain much conservatism, are almost replicated, as shown in Fig. 1. The tradeoff curve for a linear $\varphi_{\ell}$ still includes considerable conservatism. The flexibility of our SOS approach allows to gradually increase the order of $\varphi_{\ell}$ to reduce conservatism in the results, as Fig. 1 clearly shows.

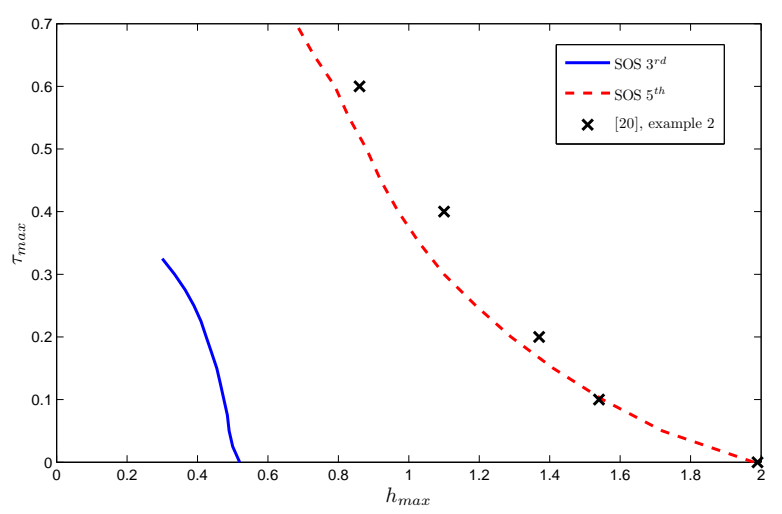

Fig. 1. Tradeoff curves for sampled data NCS.

To illustrate the form of Lyapunov functions used and to indicate some numerical values, let us provide them for the situation $\left(h_{\max }, \tau_{\max }\right)=(0.45,0.1)$. The stability of this situation is proven by a third order polynomial Lyapunov function as in (33) consisting of a linear function $\varphi_{\ell}$ and quadratic $\tilde{W}_{\ell}$ given by the SOS program as

$$
\begin{aligned}
V_{0}(\chi, \tau)= & 49.187 x+3.885 \tau x^{2}-0.340 \tau e x+52.275 e^{2} \\
& -105.470 \tau e^{2}+121.670 s^{2}-69.402 \tau s^{2} \\
V_{1}(\chi, \tau)= & 49.187 x+31.165 \tau x^{2}-18.355 \tau e x+61.087 e^{2} \\
& -132.550 \tau e^{2}-56.680 \tau x s+112.200 e s \\
& -134.830 \tau e s+55.747 s^{2}-30.193 \tau s^{2}
\end{aligned}
$$

Note that although setting $h_{\min }=\tau_{\min }=0$ was chosen for reasons of comparison to earlier work, in many practical situations there exists a nonzero lower bound due to hardware limitations. If we assume that the transmission frequency of the network cannot exceed $10 \mathrm{~Hz}$ (i.e. $\left.h_{\text {min }}=0.1\right)$ and the minimum delay is $10 \mathrm{~ms}\left(\tau_{\text {min }}=\right.$ 0.01) then new tradeoff curves in Fig. 1 can be computed. To provide some indication of how these non-zero lower bounds can lead to larger $h_{\max }, \tau_{\max }$ guaranteeing robust stability, we focus on the point $\left(h_{\max }, \tau_{\max }\right)=$ $(1.54,0.1)$, which lies on the SOS $5^{\text {th }}$ order curve in Fig. 1 (and thus corresponds to $h_{\min }=\tau_{\min }=0$ ). Adopting the nonzero lower bounds $h_{\min }=0.1$ and $\tau_{\min }=0.01$, this point becomes $(1.85,0.1)$, which is a $20 \%$ increase in robustness, in terms of $h_{\max }$, with respect to the case of zero lower bounds. This clearly shows the benefit of being able to include non-zero lower bounds on delays and transmission intervals in the analysis. While our method can easily exploit non-zero bounds, several existing methods cannot. 


\subsection{Example 2 - Shared Communication}

In this example we consider the plant and controller given in the form (1), (2) where

$$
\begin{aligned}
\dot{x}_{p} & =\left[\begin{array}{cc}
0 & 1 \\
0 & -0.1
\end{array}\right] x_{p}+\left[\begin{array}{c}
0 \\
0.1
\end{array}\right] \hat{u}, \quad y=x_{p}, \\
u & =-\left[\begin{array}{ll}
3.75 & 11.5
\end{array}\right] \hat{y},
\end{aligned}
$$

which was studied in [40], although without a shared communication medium and, hence, without protocols. We will compare our method with the technique in [15].

We consider a shared communication medium, as well as varying transmission intervals and delays, and compute stability regions for both the RR and TOD protocols. We assume that only the two states are transmitted over the network, while $u(t)=\hat{u}(t)$ for all $t \in \mathbb{R}_{\geq 0}$. This results in the networked error $e=e_{y}$, where $y=x_{p}$. The constraints from Section 3.3.2 and Section 3.3.3 are implemented in a SOS program for the RR and TOD protocols. A third order $V_{\ell, \sigma}$, which consisted of a linear $\varphi_{\ell, \sigma} \in \mathbb{R}[\tau]$ and a quadratic $\tilde{W}_{\ell, \sigma} \in \mathbb{R}[\chi]$, and a fifth order $V_{\ell, \sigma}$, which consisted of a third order $\varphi_{\ell, \sigma}$ and a quadratic $\tilde{W}_{\ell, \sigma}$, were used. The resulting tradeoff curves for the method in [15] and our SOS method are shown in Fig. 2. The SOS method proposed in this paper clearly reduces conservatism in the obtained tradeoff curves compared to [15] when considering a third order $V_{\ell, \sigma}$, and reduced conservatism even further when considering a fifth order $V_{\ell, \sigma}$ polynomial Lyapunov candidate.

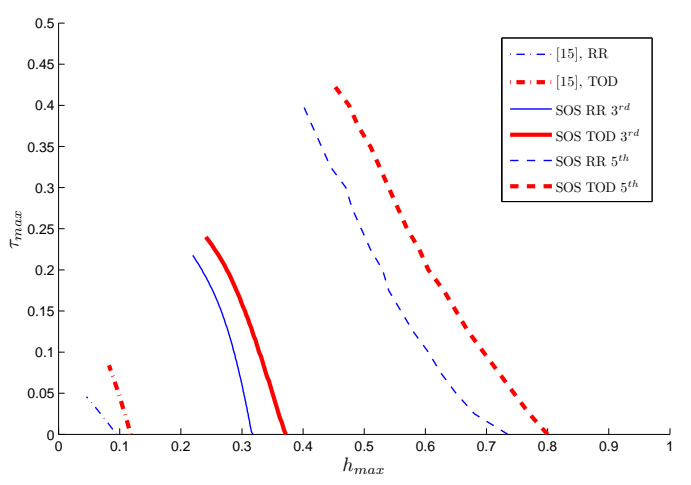

Fig. 2. Tradeoff curves for NCS with a shared communication medium with third order $V$ and fifth order $V$.

\subsection{Example 3 - Batch Reactor}

In this example the NCS benchmark system consisting the of batch reactor $[3,15,26,37]$ is compared. The batch reactor is given by a plant containing four states and a controller containing two states. For the actual system matrices, see the references provided. The two outputs $y$ of the plant share the network, implying that they cannot communicate simultaneously, and the two controlled inputs $u$ of the plant are considered wired to the controller (i.e. $u(t)=\hat{u}(t)$ for all $t \in \mathbb{R}_{>0}$ ). The results for the batch reactor are shown in Fig. $\overline{3}$ and are compared to the results of the approach in [15].

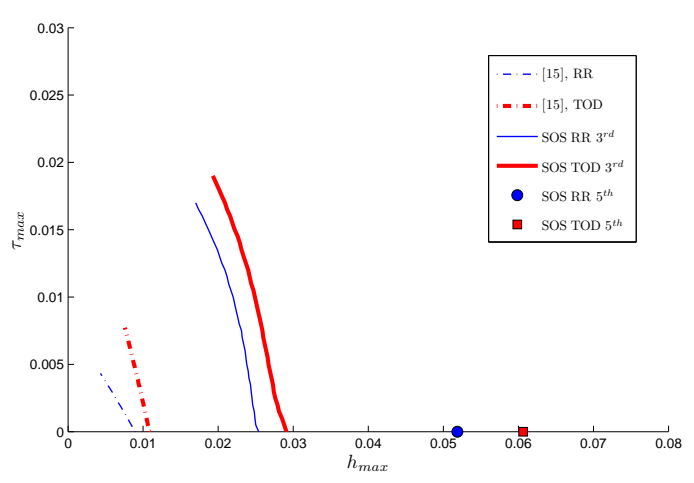

Fig. 3. Tradeoff curves for NCS benchmark batch reactor.

From Fig. 3 it can be seen that the SOS approach presented in this paper again results in less conservative results than in [15] when considering a third order $V_{\ell, \sigma}$, which consists of a linear $\varphi_{\ell, \sigma} \in \mathbb{R}[\tau]$ and quadratic $\tilde{W}_{\ell, \sigma} \in \mathbb{R}[\chi]$. We also computed a fifth order $V_{\ell, \sigma}$, which consists of a third order $\varphi_{\ell, \sigma}$ and quadratic $\tilde{W}_{\ell, \sigma}$, for the delay-free case. Interestingly, in this delay-free case, the least conservative theoretical result, until recently, for $h_{\max }$ that still guarantees stability was given in [3] as $h_{\max }=0.0108$ using the TOD protocol, while we obtain $h_{\max }=0.0606$. In [37], $h_{\max }$ was estimated (using simulations) to be between 0.06 and 0.08 for the TOD protocol. For the RR protocol, [3] provides the bound $h_{\max }=0.009$ in the delay-free case, while we obtain $h_{\max }=0.0519$. Only recently, based on a new linear discrete-time approach, [8] achieved $h_{\max }=0.066$ for the TOD protocol and $h_{\max }=0.064$ for the RR protocol in the delay-free case. These results are comparable to the results calculated with our SOS-based analysis by just using a $5^{t h}$ order Lyapunov function. Hence, using the SOS-based approach, results are obtained which are close to the available upper bounds on $h_{\max }$, while, at the same time, offering several additional beneficial features mentioned in the introduction. For instance, robust stability of the example in the next section cannot be assessed by [8].

The computational burden of the SOSP based on a fifth order polynomial Lyapunov function for NCSs including delays becomes rather high and these results are therefore not provided. This indicates the only drawback of the proposed method: the computational complexity might grow quickly with the state dimension and the order of the Lyapunov function. Improved solvers for SOS programs are needed to reduce the computation time for such complex problems. 


\subsection{Example 4-Polynomial Sampled-Data System}

In this example we will show that our method can also prove GAS for NCSs with the plant having polynomial dynamics. The system we consider is given by (1), (2) where $\dot{x}_{p}=x_{p}^{3}+x_{p}^{2} \hat{u}, y=x_{p}$ and $u=-5 \hat{y}$. The network effects we consider for this example are varying transmission intervals and delays (the communication medium is not shared). In fact, we take $h_{\min }=\tau_{\min }=0$ and aim at determining values for $\tau_{\max }$ and $h_{\max }$ for which the NCS is GAS.

To do so, the constraints from Section 3.3.1 are implemented in a SOS program. For linear plants and controllers the order of $\tilde{W}_{\ell} \in \mathbb{R}[\chi]$ in (33) is often taken to be 2 , but for this nonlinear system, higher order terms are needed. Therefore we specify the order of $\tilde{W}_{\ell}$ to be 6. In addition, we specify that the function $\varphi_{\ell} \in \mathbb{R}[\tau]$ is linear. This results in the tradeoff curves as provided in Fig. 4, showing indeed that we can analyze a NCS with a polynomial plant and controller in a systematic manner.

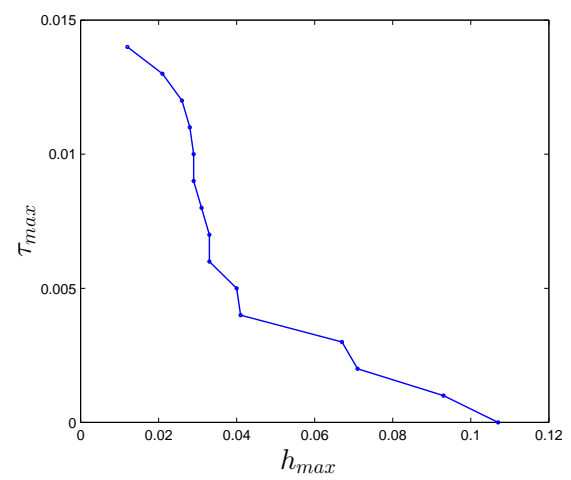

Fig. 4. Tradeoff curves for NCS with polynomial plant dynamics.

\section{Conclusions}

In this paper we have presented a sum of squares (SOS) approach for the stability analysis of NCSs that exhibit varying delays, varying transmission intervals and a shared communication medium. The NCS was modeled as a hybrid system, which allows for general continuoustime piecewise polynomial plant and controller dynamics. In order to use SOS techniques, the flow and jump map of the hybrid system were transformed into piecewise polynomial descriptions. This transformation was explicitly shown for three important cases consisting of a pure sampled-data system without a shared communication medium, and NCSs with a shared communication medium and using either the Try-Once-Discard (TOD) or Round Robin (RR) protocol. We were able to show that by using SOS techniques, it is possible to improve existing results in the literature significantly. As expected, increasing the order of the polynomial Lyapunov functions leads to improved bounds on the delays and transmission intervals (at the cost of higher computational complexity). Next to a reduction in conservatism, our method offers various other beneficial features, such as dealing with non-zero lower bounds on varying delays and transmission intervals, dealing with nonlinear (polynomial) plants and controllers, not requiring an overapproximation of the NCS (as in the discrete-time approach) and finally, offering an automated method to tackle the stability problem for NCS including varying delays, transmission intervals and a shared communication medium. The only drawback of the proposed method is that the computational complexity grows quickly with the state dimension and the order of the Lyapunov function. Improved solvers for SOS programs are needed to reduce the computation time for such complex problems.

\section{References}

[1] N.W. Bauer, P.J.H. Maas, and W.P.M.H. Heemels. Stability analysis of networked control systems: A sum of squares approach. In Proceedings of the 49th IEEE Conference on Decision and Control, December 2010.

[2] S. Boyd, L. El Ghaoui, E. Feron, and V. Balakrishnan. Linear Matrix Inequalities in System and Control Theory, volume 15 of Studies in Applied Mathematics. SIAM, Philadelphia, PA, June 1994.

[3] D. Carnevale, A. R. Teel, and D. Nešić. A Lyapunov proof of an improved maximum allowable transfer interval for networked control systems. IEEE Transactions on Automatic Control, 52(5):892-897, May 2007.

[4] A. Chaillet and A. Bicchi. Delay compensation in packetswitching networked controlled systems. In Proceedings of the 47th IEEE Conference on Decision and Control, pages 3620-3625, December 2008.

[5] G. Chesi. LMI techniques for optimization over polynomials in control: A survey. IEEE Transactions on Automatic Control, 55(11):2500-2510, November 2010.

[6] M.B.G. Cloosterman, L. Hetel, N. van de Wouw, W.P.M.H Heemels, J. Daafouz, and H. Nijmeijer. Controller synthesis for networked control systems. Automatica, 46(10):15841594,2010 .

[7] M.B.G. Cloosterman, N. van de Wouw, W.P.M.H. Heemels, and H. Nijmeijer. Stability of networked control systems with uncertain time-varying delays. IEEE Transactions on Automatic Control, 54(7):1575-1580, July 2009.

[8] M.C.F. Donkers, W.P.M.H. Heemels, N. van de Wouw, and L. Hetel. Stability analysis of networked control systems using a switched linear systems approach. Transactions on Automatic Control, 56(9):2101-2115, Sep. 2011.

[9] E. Fridman, A. Seuret, and J.P. Richard. Robust sampleddata stabilization of linear systems: an input delay approach. Automatica, 40(8):1441-1446, 2004.

[10] H. Gao, T. Chen, and J. Lam. A new delay system approach to network-based control. Automatica, 44(1):39-52, 2008.

[11] M. Garcia-Rivera and A. Barreiro. Analysis of networked control systems with drops and variable delays. Automatica, 43(12):2054-2059, 2007. 
[12] R.H. Gielen, S. Olaru, M. Lazar, W.P.M.H. Heemels, N. van de Wouw, and S. Niculescu. On polytopic inclusions as a modeling framework for systems with time-varying delays. Automatica, 46(3):615-619, 2010.

[13] R. Goebel, R. Sanfelice, and A. Teel. Hybrid dynamical systems. IEEE Control Systems Magazine, 29(2):28-93, April 2009.

[14] W.P.M.H. Heemels, D. Nesic, A.R. Teel, and N. van de Wouw. Networked and quantized control systems with communication delays. In Proceedings of the 48th IEEE Conference on Decision and Control, held jointly with the 28th Chinese Control Conference, pages 7929-7935, December 2009.

[15] W.P.M.H. Heemels, A.R. Teel, N. van de Wouw, and D. Nešić. Networked control systems with communication constraints: Tradeoffs between transmission intervals, delays and performance. IEEE Transactions on Automatic Control, 55(8):1781-1796, August 2010.

[16] W.P.M.H. Heemels, N. van de Wouw, R.H. Gielen, M.C.F. Donkers, L. Hetel, S. Olaru, M. Lazar, J. Daafouz, and S. Niculescu. Comparison of overapproximation methods for stability analysis of networked control systems. In HSCC '10: Proceedings of the 13th ACM international conference on Hybrid systems: computation and control, pages 181-190, New York, NY, USA, 2010. ACM.

[17] D. Henrion and A. Garulli. Positive Polynomials in Control, volume 312 of Lecture Notes in Control and Information Sciences. Springer-Verlag, Berlin, Heidelberg, 2005.

[18] L. Hetel, J. Daafouz, and C. Iung. Stabilization of arbitrary switched linear systems with unknown time-varying delays. IEEE Transactions on Automatic Control, 51(10):1668-1674, October 2006.

[19] M. Laurent. Sums of squares, moment matrices and optimization over polynomials. In Emerging Applications of Algebraic Geometry, volume 149 of IMA Volumes in Mathematics and its Applications, pages 157-270. Springer, 2009.

[20] K. Liu and E. Fridman. Stability analysis of networked control systems: A discontiuous Lyapunov functional approach. In Proceedings of the 48th IEEE Conference on Decision and Control, held jointly with the 28th Chinese Control Conference, pages 1330-1335, December 2009.

[21] L. Mirkin. Some remarks on the use of time-varying delay to model sample-and-hold circuits. IEEE Transactions on Automatic Control, 52(6):1109-1112, June 2007.

[22] P. Naghshtabrizi, J.P. Hespanha, and A.R. Teel. On the robust stability and stabilization of sampled-data systems: A hybrid system approach. In Proceedings of the 45 th IEEE Conference on Decision and Control, pages 4873-4878, December 2006.

[23] P. Naghshtabrizi, J.P. Hespanha, and A.R. Teel. Stability of delay impulsive systems with application to networked control systems. In Proceedings of the 2007 American Control Conference, pages 4899-4904, July 2007.

[24] P. Naghshtabrizi, J.P. Hespanha, and A.R. Teel. Exponential stability of impulsive systems with application to uncertain sampled-data systems. Systems and Control Letters, 57(5):378-385, 2008.

[25] D. Nešić and D. Liberzon. A unified framework for design and analysis of networked and quantized control systems. IEEE Transactions on Automatic Control, 54(4):732-747, April 2009.
[26] D. Nešić and A.R. Teel. Input-output stability properties of networked control systems. IEEE Transactions on Automatic Control, 49(10):1650-1667, October 2004.

[27] D. Nešić and A.R. Teel. Input-to-state stability of networked control systems. Automatica, 40(12):2121-2128, 2004.

[28] D. Nešić and A.R. Teel. Input-to-state stability of networked control systems. Automatica, 40(12):2121-2128, 2004.

[29] A. Papachristodoulou and S. Prajna. A tutorial on sum of squares techniques for systems analysis. In Proceedings of the 2005 American Control Conference, pages 2686-2700 vol. 4, June 2005.

[30] A. Papachristodoulou and S. Prajna. Robust stability analysis of nonlinear hybrid systems. IEEE Transactions on Automatic Control, 54(5):1035-1041, May 2009.

[31] P.A. Parrilo. Structured Semidefinite Programs and Semialgebraic Geometry Methods in Robustness and Optimization. Ph.D. Thesis, California Institute of Technology, Pasadena, California, May 2000.

[32] I.G. Polushin, P.X. Liu, and C.-H. Lung. On the modelbased approach to nonlinear networked control systems. Automatica, 44(9):2409-2414, 2008.

[33] S. Prajna, A. Papachristodoulou, P. Seiler, and P. A. Parrilo. SOSTOOLS: Sum of squares optimization toolbox for $M A T L A B, 2004$.

[34] J. Skaf and S. Boyd. Analysis and synthesis of statefeedback controllers with timing jitter. IEEE Transactions on Automatic Control, 54(3):652-657, March 2009.

[35] Y.S. Suh. Stability and stabilization of nonuniform sampling systems. Automatica, 44(12):3222-3226, 2008.

[36] N. van de Wouw, P. Naghshtabrizi, M.B.G. Cloosterman, and J.P. Hespanha. Tracking control for sampled-data systems with uncertain time-varying sampling intervals and delays. International Journal of Robust and Nonlinear Control, 20(4):387-411, March 2010.

[37] G.C. Walsh, H. Ye, and L.G. Bushnell. Stability analysis of networked control systems. IEEE Transactions on Control Systems Technology, 10(3):438-446, May 2002.

[38] M. Yu, L. Wang, and T. Chu. Sampled-data stabilisation of networked control systems with nonlinearity. IEEE Proceedings of Control Theory and Applications, 152(6):609614, November 2005.

[39] L. Zhang, Y. Shi, T. Chen, and B. Huang. A new method for stabilization of networked control systems with random delays. IEEE Transactions on Automatic Control, 50(8):1177-1181, August 2005.

[40] W. Zhang, M.S. Branicky, and S.M. Phillips. Stability of networked control systems. IEEE Control Systems, 21(1):8499, feb 2001. 